\title{
ADMISSIBLE TRACTION BOUNDARY CONDITIONS ALONG THE CRACK LINE IN NONLOCAL ELASTICITY*
}

BY

\author{
NASIT ARI
}

Lafayette College, Easton, Pennsylvania

\begin{abstract}
Mixed boundary conditions for integro-differential systems in general lead to overdetermined problems. A specific instance of this problem is encountered in nonlocal elasticity. The ensuing consistency problem is analyzed by utilizing the asymptotic properties of eigenfunctions associated with the kernel of a Fredholm equation of the first kind. Finally as an example, a well-posed set of mixed boundary values are derived for the one-dimensional nonlocal Griffith crack problem.
\end{abstract}

1. Introduction. In the nonlocal theory of elasticity the stress at a given point is not only a function of the strains at that particular point, but also a functional of all neighboring deformations within a nonlocal influence range $\epsilon$. See [1]. This builtin interaction structure enriches the continuum formulation by providing additional modes of response behavior. It also incorporates an internal characteristic length and extends the scope of nonlocal elasticity into the discrete substratum of material bodies. This enrichment, however, comes at the expense of rather complicated integro-differential field and boundary conditions.

The interaction between neighboring points persists also at the material surfaces. The imposition of independent mixed boundary values in adjoining regions hence leads to overdetermined (nonconsistent) problems. No solutions exist, in general, for such systems.

The difficulty with the formulation of well-posed boundary value problems for integro-differential systems is typical and it occurs frequently. A well-known example is the question of additional boundary constraints in crystal optics with spatial dispersion [2]. There, a specific integro-differential system is reduced to a higher-order differential equation. The solution, however, now contains additional integration constants. In order to determine these extra constants the solution of the differential equation is substituted back into the original integro-differential system. The consistency requirements and/or microscopic considerations then specify the needed additional boundary conditions and consequently a unique solution is picked.

${ }^{*}$ Received June 16, 1987.

(C) 1988 Brown University 
In nonlocal elasticity, too, we have to impose constraints emanating from the physics of the problem to determine the class of admissible boundary values. Here the problem is the reconciliation of two opposing dictates.

1) The applied forces should be independent from any material properties of the body, including its nonlocality.

2) On the other hand, the nonlocal boundary conditions should reflect the interdependency of adjacent boundary values and the specific nonlocality.

In order to resolve this dilemma we have to recognize that one can no longer identify the applied forces with the boundary tractions as directly as the classical theory of elasticity does. There should be a more general relationship between the applied forces and prescribed boundary tractions and it should take into account the nonlocal effects. In the limit as nonlocal interactions vanish, however, the boundary conditions should revert to their classical counterparts.

The question of existence in nonlocal mixed boundary value problems was addressed earlier by Eringen [3]. He introduced a geometry induced cut-off in the range of nonlocal kernels to avoid incompatible boundary conditions. In the present work, instead, we focus on the generation of consistent boundary conditions. To this end, we discuss admissible modifications of local boundary conditions and investigate the consequences of general asymptotic requirements on the selection of boundary values.

In Section 2 we provide a brief introduction to the fundamentals of the linear theory of nonlocal elasticity. The specific difficulties associated with nonlocal mixed boundary conditions are presented in Section 3. In Section 4 we determine the eigenfunctions of a specific nonlocal kernel and, utilizing their asymptotic properties, we obtain well-posed boundary conditions for a one-dimensional nonlocal Griffith crack problem. The results and possible generalizations of the present method are discussed in Section 5.

2. Nonlocal theory of linear elasticity. For a linear, homogeneous, and isotropic solid the constitutive equation for the nonlocal stress tensor $t_{k l}^{N}$ can be found in [1].

$$
t_{k l}^{N}(\mathbf{x})=\int \alpha\left(\left|\mathbf{x}^{\prime}-\mathbf{x}\right| ; \epsilon\right)\left[\lambda_{k l} e_{r r}\left(\mathbf{x}^{\prime}\right)+2 \mu e_{k l}\left(\mathbf{x}^{\prime}\right)\right] d V\left(\mathbf{x}^{\prime}\right)
$$

where the integral is over a three-dimensional region $V$ and $e_{k l}$ is the strain tensor derived from the displacement vector $u_{k}$ by

$$
e_{k l}=\frac{1}{2}\left(u_{k, l}+u_{l, k}\right) \text {, }
$$

$(\lambda, \mu)$ are the Lamé constants, and $\delta_{k l}$ is the Kronecker delta. Here and throughout this paper, an index following a comma represents a gradient, e.g.,

$$
u_{k, l}=\partial u_{k} / \partial x_{l}
$$

where free indices $(k, l)$ take the values $(1,2,3)$ and repeated indices are summed over the same range.

The nonlocality of the material body is characterized by the nonlocal kernel $\alpha\left(\left|\mathbf{x}^{\prime}-\mathbf{x}\right| ; \epsilon\right)$ (influence function). $\epsilon$ represents the effective range of nonlocal interactions and it could be identified with a microstructural length parameter. Both 
the functional form of $\alpha$ and the magnitude of the nonlocality parameter $\epsilon$ can be obtained from solid state wave dispersion experiments [1]. Hence the nonlocal formulation is free of adjustable parameters.

The detailed structure of the nonlocal kernel plays an important role in wave propagation problems. However, in nonlocal elasticity the kernel acts as a regularizer that averages and smooths stress fields. Thus in elasticity problems the range and the decay rate of nonlocal interactions suffice to define the influence kernels. This flexibility also enables one to represent the kernels in mathematically convenient forms, such as shown on Fig. 1. Note that these kernels constitute Dirac- $\delta$ sequences as $\epsilon \rightarrow 0$ and in this limit the nonlocal constitutive equations reduce to those of classical elasticity, i.e.,

$$
\lim _{\epsilon \rightarrow 0} t_{k l}^{N}=\lambda \delta_{k l} e_{r r}+2 \mu e_{k l}=t_{k l}
$$

(a)

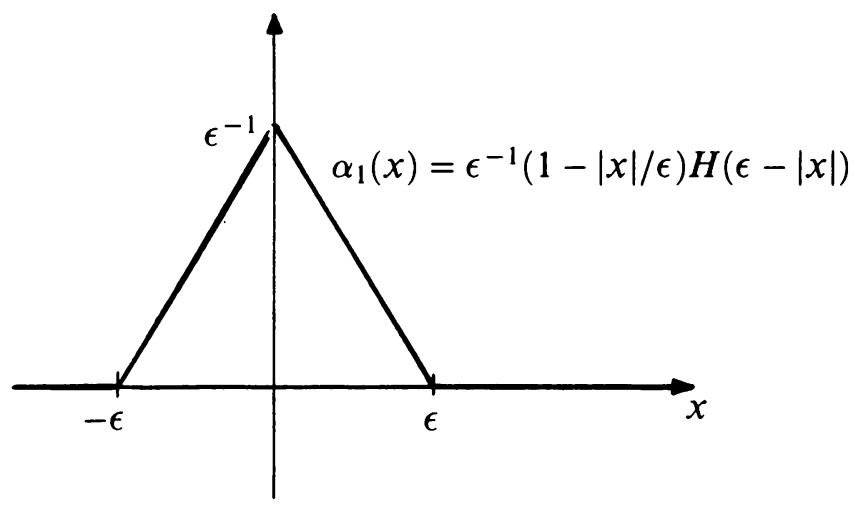

(b)

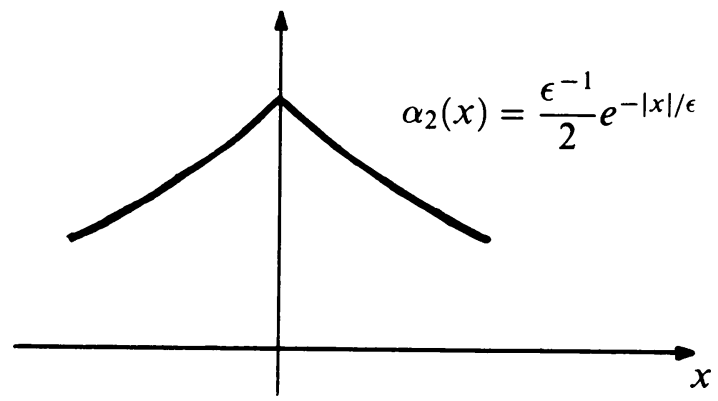

FIG. 1. Nonlocal kernels

When body and inertia forces are absent the equilibrium equations are given by

$$
t_{k l, k}^{N}=0
$$

and substitution of (2.1) into (2.4) yields the nonlocal field equations.

At this point the only apparent difference from classical elasticity is the functional form of (2.1). However, the introduction of nonlocal interdependency requires also important modifications of boundary conditions as explained in the next section. 
3. Overdetermined mixed boundary conditions. From Fig. 1a and Eq. (2.1) we observe that the minimum distance between two material points that can deform independently is $\epsilon$, i.e., the nonlocality range. If the interaction range is not finite such as on Fig. 1b we can still define an effective cut-off distance.

To illustrate the effect of the interactions on mixed boundary value problems, let us denote the part of the boundary where stresses and displacements are prescribed by $S_{t}$ and $S_{u}$ respectively (see Fig. 2). By using the kernel shown on Fig. 1a we observe that near the common border of the $S_{t}$ and $S_{u}$ regions there will always be an interfusion region $S_{i}$ that will be both in $S_{t}$ and $S_{u}$. Thus it will be impossible to prescribe stress conditions on $S_{t} \cap S_{i}$ that are independent of displacement conditions in $S_{u}$.

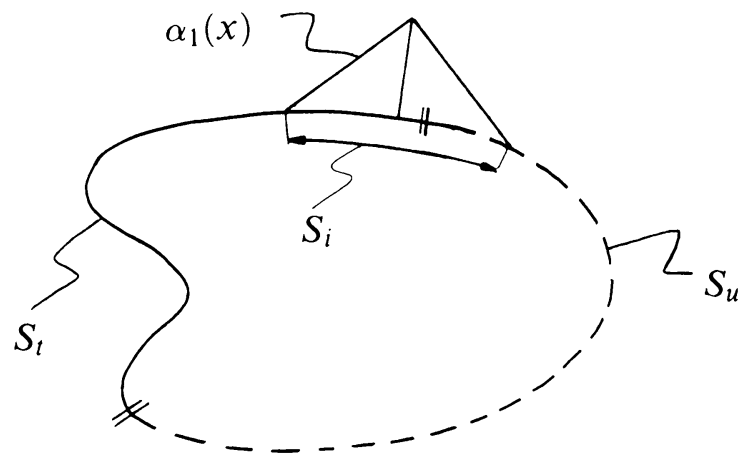

FIG. 2. Interfusion region

One way out of this dilemma is to impose a separation region between $S_{t}$ and $S_{u}$ where the boundary conditions are left undetermined. (This approach is similar to a discrete, solid-state picture of the problem, and it will be the subject of a forthcoming paper.) The other route is to accept the existence of this overlapping region but to eliminate the overdetermination by continuity requirements.

In the present work, we follow the second route and investigate the consequences of two general asymptotic requirements on the selection of well-posed boundary values. Specifically we assume the following.

1) In the local limit (i.e., as $\epsilon \rightarrow 0$ ) the nonlocal boundary conditions should reduce to the mixed boundary conditions of classical elasticity.

2) The transition from the local to the nonlocal state should produce only smooth changes in the deformation fields, i.e., the displacements should be analytic functions of the nonlocality parameter $\epsilon$.

In the next section, an explicit implementation of this algorithm for the one-dimensional Griffith crack problem yields a unique set of controllable stress and displacement conditions along the crack line.

4. Derivation of the modified boundary conditions. The nonlocal one-dimensional Griffith crack problem in [4] is defined by the field equations

$$
\begin{aligned}
\gamma^{2} v_{x x}+v,_{y y} & =0, \quad y>0, \\
\gamma^{2} & =\mu /(\lambda+2 \mu),
\end{aligned}
$$


and the boundary conditions

$$
\begin{aligned}
& t_{y y}^{N}(x, 0)=-t_{0}, \quad|x|<l, \quad x \in S_{t}, \\
& v(x, 0)=0, \quad|x| \geq l, \quad x \in S_{u} .
\end{aligned}
$$

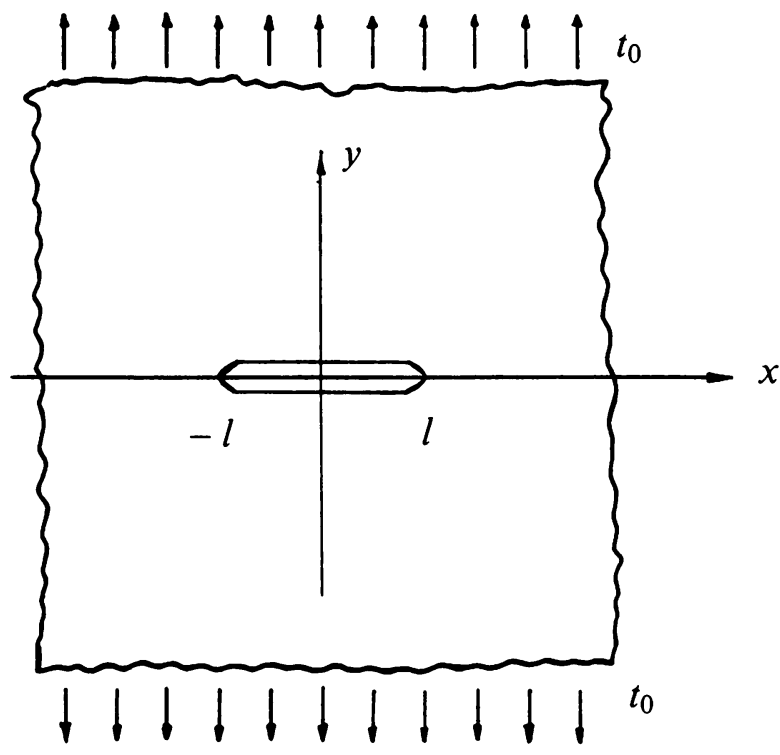

Fig. 3. Griffith crack

As shown on Fig. 3,v is the displacement along the $y$-direction and $t_{y y}^{N}$ denotes the normal nonlocal stress. $t_{0}$ is the uniform applied tension at $y= \pm \infty$. In the one-dimensional case (2.1) reduces to

$$
t_{y y}^{N}=\int_{-\infty}^{\infty} \alpha\left(\left|x^{\prime}-x\right| ; \epsilon\right)(\lambda+2 \mu) v_{y}\left(x^{\prime}, y\right) d x^{\prime} .
$$

The boundary conditions (4.2-4.3) are carried over from classical elasticity and as such the imposed stress and displacements are independent of any nonlocality parameters. On the other hand, in Sec. 3 we have shown that for self-consistency the actual boundary conditions have to be modified to reflect the nonlocal interdependencies. To this end, we generalize the stress boundary condition (4.2) to

$$
t_{y y}^{N}(x, 0)=-t_{0}+t_{M}\left(x ; \alpha, \epsilon, t_{0}\right)=T^{N}(x ; \epsilon), \quad|x|<l,
$$

where $t_{M}$ is the additional boundary stress due to nonlocal response of the body to the boundary tractions. It depends on the nonlocal kernel and applied forces. With the addition of $t_{M}$ the actual boundary condition is given by $T^{N}(x ; \epsilon)$, which is now expected to be consistent with the imposed displacement boundary condition (4.3). In the limit, as $\epsilon \rightarrow 0, t_{M}$ should vanish and we should recover the classical stress boundary condition.

In the sequel we illustrate the determination of $t_{M}$ by using the particular kernel shown on Fig. 1b, i.e.,

$$
\alpha\left(\left|x^{\prime}-x\right| ; \epsilon\right)=\left(\epsilon^{-1} / 2\right) \exp \left(-\left|x^{\prime}-x\right| / \epsilon\right) .
$$


Introducing the Fourier representation

$$
v(x, y)=(2 / \pi)^{1 / 2} \int_{0}^{\infty} A(k, \epsilon) e^{\gamma k y} \cos k x d k
$$

and substituting it into (4.1-4.6), we reduce the mixed boundary value problem to the solution of a pair of dual integral equations

$$
\begin{gathered}
A_{0} \int_{0}^{\infty} k \frac{A(k)}{1+\epsilon^{2} k^{2}} \cos k x d k=-t_{0}+t_{M}=T^{N}(x ; \epsilon), \quad x \leq l, \\
\int_{0}^{\infty} A(k) \cos k x d k=0, \quad x>l, \\
A_{0}=-\gamma(\lambda+2 \mu)(2 / \pi)^{1 / 2} .
\end{gathered}
$$

These typical dual equations can further be transformed to a single Fredholm equation by standard methods, i.e., the ansatz

$$
A(k)=A_{0}^{-1} \int_{0}^{1} g(t) J_{0}(k l t) d t
$$

satisfies (4.9) automatically and (4.8) becomes

$$
\int_{0}^{1} d t g(t) \int_{0}^{\infty} k \frac{J_{0}(k l t)}{1+\epsilon^{2} k^{2}} \cos k x d k=T^{N}(x ; \epsilon), \quad|x| \leq l .
$$

Applying the integration operator

$$
(2 / \pi) \int_{0}^{z} d x\left(z^{2}-x^{2}\right)^{-1 / 2}
$$

to (4.11) we obtain finally

$$
\int_{0}^{1} S(z, t) g(t) d t=(2 / \pi) \int_{0}^{z} \frac{T^{N}(x ; \epsilon)}{\left(z^{2}-x^{2}\right)^{1 / 2}} d x=f(z)
$$

where

$$
\begin{aligned}
S(z, t) & =\varepsilon^{-2} \begin{cases}K_{0}(t / \varepsilon) I_{0}(z / \varepsilon), & z<t, \\
K_{0}(z / \varepsilon) I_{0}(t / \varepsilon), & z>t,\end{cases} \\
\varepsilon & =\epsilon / l,
\end{aligned}
$$

and $K_{0}, I_{0}$ are modified Bessel functions of zeroth order.

The selection problem can now be expressed as a concise mathematical question:

Given a nonlocal kernel and hence $S(z, t)$ of the Fredholm equation of the first kind in (4.13), which classes of $T^{N}(x ; \varepsilon)$ will yield a solution $g(t)$ of class $L_{2}$.

Fortunately, the Hilbert-Schmidt theorem for symmetric Fredholm equations of the first kind provides a complete discussion of the solutions to (4.13) in $L_{2}$-spaces ([5], pp. 143-144). The Fredholm Theory first requires the solution of an associated eigenfunction problem

$$
\int_{0}^{1} S(z, t) \phi_{n}(t) d t=\phi_{n}(z) .
$$


Then both $g(t)$ and $f(z)$ of (4.13) can be represented as a series in the eigenfunctions $\phi_{n}$ and the solution of (4.13) is

$$
g(t)=\sum g_{n} \phi_{n}(t), \quad g_{n}=\lambda_{n} f_{n}
$$

where

$$
f_{n}=\int_{0}^{1} f(t) \phi_{n}(t) d t
$$

For $g(t)$ to be in $L_{2}$, however, the Riesz-Fischer Theorem requires that the following infinite series converge:

$$
\sum g_{n}^{2}=\sum \lambda_{n}^{2} f_{n}^{2}
$$

In order to obtain the eigenvalues $\lambda_{n}$ and eigenfunctions $\phi_{n}$ we apply the operator

$$
Q=\frac{d^{2}}{d t^{2}}+\frac{1}{t} \frac{d}{d t}-\varepsilon^{-2}
$$

to $(4.13)$, and noting that

$$
\begin{aligned}
Q\left[K_{0}(t / \varepsilon)\right] & =-\varepsilon^{-2} \delta(t) / t \\
Q\left[I_{0}(t / \varepsilon)\right] & =0
\end{aligned}
$$

$(\delta(t)$ is the Dirac-delta measure), we transform (4.15) into an equivalent differential equation:

$$
\frac{d^{2}}{d t^{2}} \phi+t^{-1} \frac{d \phi}{d t}+\varepsilon^{-2}\left(\lambda t^{-1}-1\right) \phi=0
$$

The solutions to (4.21) can be found in ([6], p. 33),

$$
\phi(t)=t^{-1 / 2} P_{\mathscr{K}, 0}(2 t / \varepsilon), \quad \mathscr{K}=\lambda /(2 \varepsilon),
$$

where $P_{\mathscr{K}, 0}$ denotes $M_{\mathscr{K}, 0}$ or $W_{\mathscr{K}, 0}$, both related to Whittaker's differential equation. Since $t^{-1 / 2} W_{\mathscr{K}, 0}(t)$ has a logarithmic singularity at the origin ([6], p. 28), $g(t)$ will be a linear combination of only the eigenfunctions,

$$
\begin{aligned}
\phi_{n} & =q_{n} t^{-1 / 2} M_{\mathscr{K}_{n}, 0}(2 t / \varepsilon) \\
& =q_{n}(2 / \varepsilon)^{1 / 2} e^{t / \varepsilon}{ }_{1} F_{1}\left(1 / 2+\mathscr{K}_{n} ; 1 ;-2 t / \varepsilon\right),
\end{aligned}
$$

where ${ }_{1} F_{1}$ is the confluent hypergeometric function and the orthonormalization constant $q_{n}$ is defined by

$$
q_{n}^{-2}=\int_{0}^{1} t^{-1} M_{\mathscr{T}_{n}, 0}^{2}(2 t / \varepsilon) d t
$$

The eigenvalues $\lambda_{n}=2 \varepsilon \mathscr{K}_{n}$ can be determined by substituting (4.23) into (4.15). The resulting eigenvalue condition is at $t=2 / \varepsilon$ :

$$
M_{\mathscr{H}_{n}, 0}(t) W_{0,0}(t)-W_{\mathscr{H}_{n}, 0}(t) M_{0,0}(t)=0
$$

Using (4.25) and the asymptotic expansions of $M_{\mathscr{K}, 0}$ for large $\mathscr{K}_{n}$ ([6], p. 97),

$$
M_{\mathscr{K}, 0}(z) \sim(z / \mathscr{K})^{1 / 4} \cos \left[2(z \mathscr{K})^{1 / 2}-\pi / 4\right] \cdot\left\{1+O\left(|\mathscr{K}|^{-1 / 2}\right)\right\}, \quad \mathscr{K} \rightarrow \infty,
$$

we find that as $n \rightarrow \infty$ the eigenvalues behave as ${ }^{1}$

$$
\lambda_{n} \sim O\left(n^{2}\right), \quad n \rightarrow \infty .
$$

\footnotetext{
${ }^{1}$ The result (4.27) and the orthogonality and completeness of the eigenfunctions $\phi_{n}$ is due to the SturmLiouville character of the present eigenvalue problem.
} 
Hence the $L_{2}$-existence condition now requires that the series

$$
\sum n^{4} f_{n}^{2}
$$

converge.

In order to investigate the case $t_{M}=0, T^{N}=-t_{0}$ we first compute $f(z)$ in (4.13) and find $f(z)=-t_{0}$. Consequently

$$
f_{n}=-t_{0} \int_{0}^{1} q_{n} t^{-1 / 2} M_{\mathscr{K}_{n}, 0}(2 t / \varepsilon) d t
$$

By utilizing (4.26) one can show that

$$
q_{n} \sim O\left(\mathscr{K}_{n}^{1 / 4}\right)
$$

and

$$
f_{n} \sim O\left(\mathscr{K}_{n}^{-1 / 2}\right) .
$$

Since $f_{n}^{2} \sim O\left(n^{-2}\right)$ the series (4.28) does not converge and there will be no $L_{2}$-solution if the boundary condition (4.2) is identical to the classical one. The same procedure will show that there will be no $L_{2}$-solution if $T^{N}(x, \varepsilon)$ is a polynomial independent of $\varepsilon$.

These mathematical results are as expected from our discussion in Sec. 3. Next we turn to the more difficult problem of determining the additional boundary traction $t_{M}(x ; \varepsilon)$.

A formal solution to the pair of dual integral equations can be found as

$$
A(k)=\int_{0}^{1} h(t) J_{0}(k l t) d t
$$

where

$$
h(t)=A_{0}^{-1}(2 / \pi) t \int_{0}^{t} \frac{\left(1-\varepsilon^{2} \partial^{2} / \partial x^{2}\right) T^{N}(x ; \varepsilon)}{\left(t^{2}-x^{2}\right)^{1 / 2}} d x .
$$

For a uniform applied load,

$$
T^{N}(x ; \varepsilon)=-t_{0}+t_{M}(x ; \varepsilon)
$$

and substitution of (4.32-4.33) into the dual equations (4.8-4.9) yields for (4.13)

$$
\int_{0}^{1} S(z, t) h(t) d t=(2 / \pi) \int_{0}^{z} \frac{t_{M}(x, \varepsilon) d x}{\left(z^{2}-x^{2}\right)^{1 / 2}}-t_{0} \varepsilon^{-1} K_{1}\left(\varepsilon^{-1}\right) I_{0}(z / \varepsilon) .
$$

The Fredholm theory requires the expansions of both $h(t)$ and the right-hand side of (4.34) leading to an Abel equation for $t_{M}(x ; \varepsilon)$ whose solution is given by

$$
t_{M}(x ; \varepsilon)=t_{0} \varepsilon^{-1} K_{1}\left(\varepsilon^{-1}\right) \cosh (x / \varepsilon)+\frac{d}{d x} \int_{0}^{x} \frac{z \sum c_{n}(\varepsilon) \phi_{n}(z) d z}{\left(x^{2}-z^{2}\right)^{1 / 2}}, \quad|x|<1 .
$$

Large $z$ asymptotic behavior of

$$
\phi(z)=q_{n} z^{-1 / 2} M_{\mathscr{K}_{n}, 0}(2 z / \varepsilon) \sim O\left(e^{z / \varepsilon} z^{-\left(\mathscr{K}_{n}+1 / 2\right)}\right)
$$


([5], p. 91) and the limit condition $t_{M}(x ; \varepsilon) \rightarrow 0$ as $\varepsilon \rightarrow 0$ now forces the undetermined coefficient $c_{n}(\varepsilon)$ to be in the form

$$
c_{n}(\varepsilon)=e^{-l / \varepsilon} p_{n}(\varepsilon)
$$

where $\left|p_{n}(\varepsilon)\right| \leq M<\infty$. The substitution of (4.36), (4.33), (4.32) into (4.7) shows that the second asymptotic requirement of Sec. 3 that $v(x ; \varepsilon)$ be analytic functions of $\varepsilon$ can now be satisfied only when $p_{n}(\varepsilon)=0$. Hence the unique $t_{M}$ is given by

$$
t_{M}(x, \varepsilon)=t_{0} \varepsilon^{-1} K_{1}\left(\varepsilon^{-1}\right) \cosh (x / \varepsilon) .
$$

In the next section we discuss the specific results obtained so far in a more general framework.

5. Discussion of the results. In Sec. 4 the controllability problem is reduced to an equivalent existence question for $L_{2}$-solutions of a Fredholm equation. In this approach, utilizing only the properties of the associated eigenfunctions, we were able to

1) decide whether a given set of boundary conditions will lead to nonconsistency;

2) modify a given set of classical boundary conditions such that they constitute a well-posed boundary condition.

Since the present approach depends only on the asymptotic characteristics of the eigenfunctions, it can be generalized to more complicated nonlocal kernels. For a class of kernels our approach is equivalent to the method of reduction of integrodifferential systems to higher-order differential equations. For example, the dual integral equations $(4.8-4.9)$ can be reduced to an easily solvable pair by utilizing the operator $\left(1-\varepsilon^{2} \partial^{2} / \partial x^{2}\right)$ to $(4.8)$, i.e.,

$$
\begin{aligned}
A_{0} \int_{0}^{\infty} k A(k) \cos k x d k & =\left(1-\varepsilon^{2} \partial^{2} / \partial x^{2}\right) T^{N}(x, \varepsilon), & & x<1, \\
\int_{0}^{\infty} A(k) \cos k x d k & =0, & & x \geq 1 .
\end{aligned}
$$

If $T^{N}(x ; \varepsilon)=-t_{0}$ then the formal solution will be

$$
A(k)=A_{0}^{-1} J_{1}(k) / k
$$

However, notice that $(5.3)$ is at the same time a solution to a large class of boundary tractions

$$
T^{N}(x, \varepsilon)=-t_{0}+A e^{x / \varepsilon}+B e^{-x / \varepsilon}
$$

where $A$ and $B$ have to be determined from consistency requirements, i.e., by substituting (5.4) and (5.3) into (4.8). That operation also yields a unique solution for $T^{N}$,

$$
T^{N} / t_{0}=-1+\varepsilon^{-1} K_{0}\left(\varepsilon^{-1}\right) \cosh (x / \varepsilon),
$$

which is identical to (4.39).

In cases where a reduction is not apparent, however, then the present eigenfunction method will still provide valuable information. The modified boundary conditions 
(5.5) and (4.3) now constitute a consistent set of boundary conditions. A comparison with the classical traction boundary conditions is shown in Fig. 4. Finally we should mention that the unique solution of the problem yields a finite stress at the crack tip, i.e.,

$$
t_{y y}(l, 0)=t_{0} \varepsilon^{-1} K_{0}\left(\varepsilon^{-1}\right) \cosh \left(\varepsilon^{-1}\right)
$$

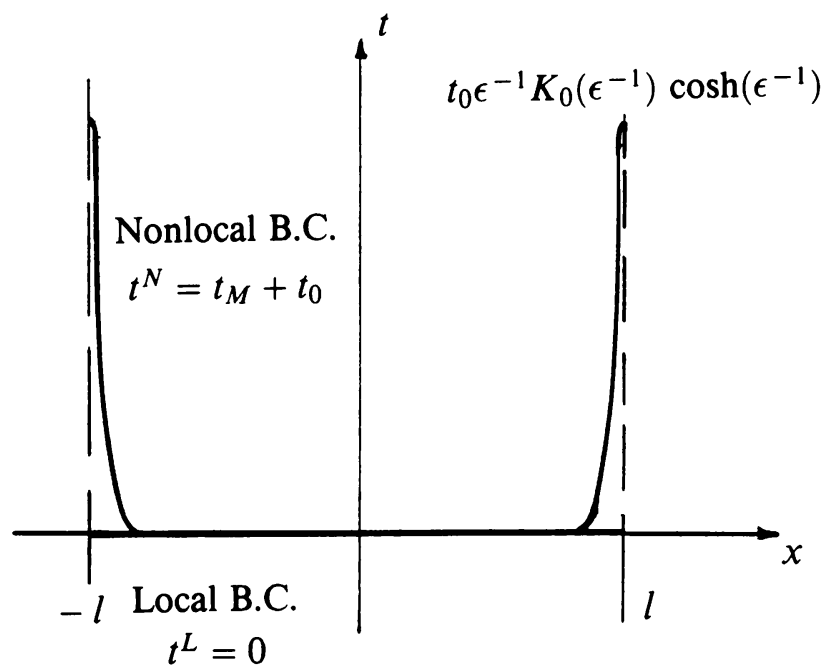

FIG. 4. Nonlocal and local boundary conditions

\section{REFERENCES}

[1] A. C. Eringen, Linear theory of nonlocal elasticity and dispersion of plane waves, Internat. J. Engrg. Sci. 10, 233-248 (1972)

[2] A. A. Maradudin and D. L. Mills, Effect of spatial dispersion on the properties of a semi-infinite dielectric, Phys. Rev. B 7, 2787-2809 (1973)

[3] A. C. Eringen, On the nature of boundary conditions for crack tip stress, Arch. Mech. 33, 937-945 (1981)

[4] A. C. Eringen, C. G. Speziale, and B. S. Kim, Crack tip problem in nonlocal elasticity, J. Mech. Phys. Solids 25, 339-355 (1977)

[5] F. G. Tricomi, Integral Equations, Interscience Pub., New York, 1957.

[6] H. Buchholz, The Confluent Hypergeometric Function, Springer-Verlag, New York, 1969 